\author{
S. V. Lukyanov, A. I. Sanzhur
}

Institute for Nuclear Research, National Academy of Sciences of Ukraine, Kyiv

\title{
NEUTRON SKIN AND HALO IN MEDIUM AND HEAVY NUCLEI WITHIN THE EXTENDED THOMAS - FERMI THEORY
}

The neutron skin and halo distributions in medium and heavy nuclei are calculated within the extended Thomas Fermi approximation. Calculations are carried out for the effective Skyrme-like forces using the direct variational method. The analytical expression for the isovector shift of the rms radii $\Delta r_{n p}$ as a sum of skin- and halo-like terms is obtained. The contribution of halo and skin terms to $\Delta r_{n p}$ are found to be approximately equal.

Keywords: neutron skin, neutron halo, Thomas - Fermi theory, variational method, Skyrme forces.

\section{Introduction}

For the analysis of the experimental data the simplest two-parameter Fermi $(2 \mathrm{pF})$ distribution of normalized nucleon densities is often used

$$
F_{q}(r)=\left[1+\exp \left(\frac{r-R_{q}}{a_{q}}\right)\right]^{-1},
$$

where $R_{q}$ is the half-density radius and $a_{q}$ is the diffuseness parameter of the distribution. Here $q=n$ is for neutron and $q=p$ for proton distributions. There are two opposite pictures in description of a two-component finite Fermi system with $2 \mathrm{pF}$. The first one is the so-called "neutron skin-type" distribution having the neutron half-density radius larger than the proton half-density radius, $R_{n}>R_{p}$, and equal diffuseness parameters $a_{n}=a_{p}$. The second one is the "neutron halo-type" distribution having $R_{n}=R_{p}$ and $a_{n}>a_{p}$. A mixture of the neutron "skin-type" and "halo-type" distributions having $R_{n}>R_{p}$ and $a_{n}>a_{p}$. is also possible. It is found that the experimental data can be reproduced by a variety of these $2 \mathrm{pF}$ distributions with different values of $R_{n}-R_{p}$ and $a_{n}-a_{p}$ [1]. In that sense there is an ambiguity in theoretical description of the experimental data.

In the present paper we study this ambiguity within the extended Thomas - Fermi approximation (ETFA) based on the direct variational method. The nucleon densities $\rho_{q}(r)$ are generated by the profile functions which are obtained from the requirement that the energy of the nucleus should be stationary with respect to variations of these profiles. The profile functions fulfill the leptodermous conditions and take into account some asymmetry of the nucleon distributions like in real nuclei.

\section{The model}

In general, the total energy of a nucleus includes the kinetic and potential energies and is given by [2 - 4]

$$
E_{\mathrm{tot}}=\int d \boldsymbol{r}\left[\epsilon_{\mathrm{kin}}(\boldsymbol{r})+\epsilon_{\mathrm{pot}}(\boldsymbol{r})\right]
$$

where the potential energy density, $\epsilon_{\mathrm{pot}}(\boldsymbol{r})$, includes the NN-interaction, $\epsilon_{\mathrm{NN}}(\boldsymbol{r})$, the spin-orbit part of the $\mathrm{NN}$-interaction, $\epsilon_{\mathrm{SO}}(\boldsymbol{r})$, and the Coulomb energy density, $\epsilon_{\mathrm{C}}(\boldsymbol{r})$

$$
\epsilon_{\mathrm{pot}}(\boldsymbol{r})=\epsilon_{\mathrm{NN}}(\boldsymbol{r})+\epsilon_{\mathrm{SO}}(\boldsymbol{r})+\epsilon_{\mathrm{C}}(\boldsymbol{r}) .
$$

In the framework of ETFA, the kinetic energy density is given by the sum of the neutron and proton contributions [3]

$$
\epsilon_{\mathrm{kin}}(\boldsymbol{r})=\epsilon_{\mathrm{kin}, \mathrm{n}}(\boldsymbol{r})+\epsilon_{\mathrm{kin}, \mathrm{p}}(\boldsymbol{r}),
$$

where

$\epsilon_{\text {kin }, \mathrm{q}}(\boldsymbol{r})=\frac{\hbar^{2}}{2 m}\left[\frac{3}{5}\left(3 \pi^{2}\right)^{2 / 3} \rho_{q}^{5 / 3}+\eta \frac{1}{36} \frac{\left(\nabla \rho_{q}\right)^{2}}{\rho_{q}}+\frac{1}{3} \nabla^{2} \rho_{q}\right]$

Here $m$ is the bare nucleon mass. The semiclassical consideration gives the value of the parameter $\eta=1$ in Eq. (5) [2, 3]. In the asymptotic limit $r \rightarrow \infty$, the semiclassical particle density $\rho_{q}$ with $\eta=1$ falls off to zero significantly faster than the one from the quantum-mechanical calculation, where one has $\eta=4$. We will use both values of the parameter $\eta$ to study the neutron halo and skin appearances and their superposition in nuclei.

For the effective nucleon-nucleon interaction we will use the Skyrme force [3]:

(C) S. V. Lukyanov, A. I. Sanzhur, 2016 


$$
\begin{aligned}
& \epsilon_{\mathrm{NN}}(\boldsymbol{r})=\frac{t_{0}}{2}\left[\left(1+\frac{x_{0}}{2}\right) \rho^{2}-\left(x_{0}+\frac{1}{2}\right)\left(\rho_{n}^{2}+\rho_{p}^{2}\right)\right]+ \\
& +\frac{1}{16}\left[3 t_{1}\left(1+\frac{x_{1}}{2}\right)-t_{2}\left(1+\frac{x_{2}}{2}\right)\right](\nabla \rho)^{2}- \\
& +\frac{t_{3}}{12} \rho^{v}\left[\left(1+\frac{x_{3}}{2}\right) \rho^{2}-\left(x_{3}+\frac{1}{2}\right)\left(\rho_{n}^{2}+\rho_{p}^{2}\right)\right]+ \\
& +\frac{1}{4}\left[t_{1}\left(1+\frac{x_{1}}{2}\right)+t_{2}\left(1+\frac{x_{2}}{2}\right)\right] \epsilon_{k i n} \rho+ \\
& -\frac{1}{16}\left[3 t_{1}\left(x_{1}+\frac{1}{2}\right)+t_{2}\left(x_{2}+\frac{1}{2}\right)\right]\left[\left(\nabla \rho_{n}\right)^{2}+\left(\nabla \rho_{p}\right)^{2}\right] \text {, }
\end{aligned}
$$$$
+\frac{1}{4}\left[t_{2}\left(x_{2}+\frac{1}{2}\right)-t_{1}\left(x_{1}+\frac{1}{2}\right)\right]\left(\epsilon_{k i n, n} \rho_{n}+\epsilon_{k i n, p} \rho_{p}\right)+
$$

where $\rho=\rho_{n}+\rho_{p}$ is the total density of nucleons. The spin-orbit part of the Skyrme force is written as

$$
\epsilon_{\mathrm{SO}}(\boldsymbol{r})=-\frac{m^{*}(r)}{\hbar^{2}} \frac{W_{0}^{2}}{4}\left[\rho_{n}\left(2 \nabla \rho_{n}+\nabla \rho_{p}\right)^{2}+\rho_{p}\left(\nabla \rho_{n}+2 \nabla \rho_{p}\right)^{2}\right] .
$$

Here $t_{0}, t_{1}, t_{2}, t_{3}, x_{0}, x_{1}, x_{2}, x_{3}, v, W_{0}$ are the parameters of the Skyrme interaction and the effective mass of a nucleon is

$$
m^{*}(r)=\frac{m}{1+\beta \rho(r)},
$$

where

$$
\beta=\frac{2 m}{\hbar^{2}} \frac{1}{4}\left[\frac{1}{4}\left(3 t_{1}+5 t_{2}\right)+t_{2} x_{2}\right]
$$

At the moment there are plenty of parameter sets $t_{i}, x_{i}, v$ and $W_{0}$ for different modifications of the Skyrme interaction. They are adjusted using the well-known properties of the nuclear matter and real nuclei and denoted by letters as SI, SIII, SkM* and so on. Below we will use this kind of notifications. The Coulomb energy density is taken in the well-known form [4]

$$
\epsilon_{\mathrm{C}}(\boldsymbol{r})=e^{2} \rho_{p}(\boldsymbol{r}) \frac{1}{2} \int d \boldsymbol{r}^{\prime} \frac{\rho_{p}\left(\boldsymbol{r}^{\prime}\right)}{\left|\boldsymbol{r}-\boldsymbol{r}^{\prime}\right|}-\frac{3}{4} e^{2}\left(\frac{3}{\pi}\right)^{1 / 3} \rho_{p}^{4 / 3}(\boldsymbol{r}) .
$$

The unknown values of $\rho_{q}$ can be evaluated from the condition of equilibrium. The corresponding condition implies that the total energy $E_{\text {tot }}$ reaches a minimum value for a given number of neutrons, $N$, and protons, $Z$

$$
N=\int d r \rho_{n}(\boldsymbol{r}), Z=\int d r \rho_{p}(\boldsymbol{r}) .
$$

In general, one can use an arbitrary trial function as nucleon distribution. In any case the variational method with the effective nucleon-nucleon interaction (6) reduces the nucleon distribution to that like $2 \mathrm{pF}$ (1). Following the direct variational method [5] the trial function for $\rho_{q}(\boldsymbol{r})$ is taken as a power of the Fermi function

$$
\rho_{q}(\boldsymbol{r})=\rho_{0, q}\left[1+\exp \left(\frac{r-R_{q}}{a_{q}}\right)\right]^{-\xi},
$$

where $\rho_{0, q}, R_{q}, a_{q}$ and $\xi$ are the variational parameters. The variational parameter $\xi$ takes into account the asymmetry of the nucleon distributions around the nuclear surface.

\section{Numerical results}

The nuclear rms radius for nucleons is defined as

$$
\sqrt{r_{q}^{2}}=\sqrt{\int d \boldsymbol{r} r^{2} \rho_{q}(r) / \int d \boldsymbol{r} \rho_{q}(r)} .
$$

The difference between the neutron and proton root mean square radii gives the neutron-skin thickness

$$
\Delta r_{n p}=\sqrt{r_{n}^{2}}-\sqrt{r_{p}^{2}}
$$

From the definition of trial functions it is seen that the value of $\Delta r_{n p}$ can be described by the different radii $R_{q}$ (skin effect) and the different diffusenesses $a_{q}$ (halo effect) of neutron and proton distributions, see also [6]. Within the leptodermous approximation $a_{q} / R_{q} \ll 1$ one can separate the above contributions and the value of $\Delta r_{n p}$ is written as

$$
\Delta r_{n p}=\Delta r_{n p}^{\text {skin }}+\Delta r_{n p}^{\text {halo }}+O\left(A^{-4 / 3}\right),
$$

where $\Delta r_{n p}^{\text {skin }}$ and $\Delta r_{n p}^{\text {halo }}$ are caused by the skin and halo effects, respectively. The corresponding values 
are given by (see Appendix A)

$$
\begin{aligned}
& \Delta r_{n p}^{s k i n} \approx \sqrt{\frac{3}{5}} \Delta_{R}\left\{1+\frac{7}{2}\left[\kappa_{0}^{2}(\xi)-2 \kappa_{1}(\xi)\right]\left(\frac{a}{R}\right)^{2}-\right. \\
& \left.-\frac{1}{3}\left(75 \kappa_{0}^{3}(\xi)-204 \kappa_{0}(\xi) \kappa_{1}(\xi)+81 \kappa_{2}(\xi)\right)\left(\frac{a}{R}\right)^{3}\right\},
\end{aligned}
$$

and

$$
\begin{gathered}
\Delta r_{n p}^{\text {halo }} \approx \sqrt{\frac{3}{5}} \Delta_{a}\left\{\kappa_{0}(\xi)-7\left[\kappa_{0}^{2}(\xi)-2 \kappa_{1}(\xi)\right] \frac{a}{R}+\right. \\
\left.+\frac{1}{2}\left(75 \kappa_{0}^{3}(\xi)-204 \kappa_{0}(\xi) \kappa_{1}(\xi)+81 \kappa_{2}(\xi)\right)\left(\frac{a}{R}\right)^{2}\right\},
\end{gathered}
$$

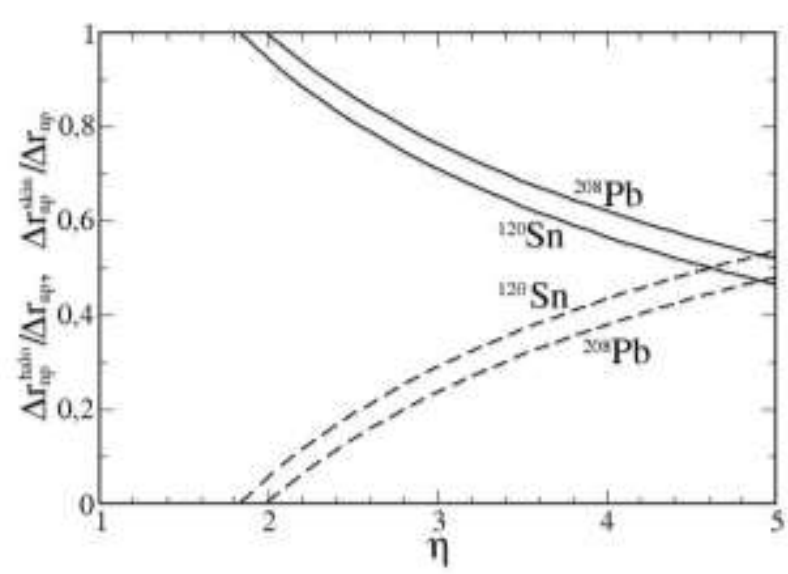

Fig. 1. The partial contribution to the isotopic shift of radii $\Delta r_{n p}$ from the skin effect, $\Delta r_{n p}^{s k i n} / \Delta r_{n p}$ (solid lines) and the halo effect, $\Delta r_{n p}^{\text {halo }} / \Delta r_{n p}$ (dashed lines) versus the parameter $\eta$. The calculations have been performed with SkM parametrization for nuclei ${ }^{120} \mathrm{Sn}$ and ${ }^{208} \mathrm{~Pb}$.

This means that the halo component is negative and reduces the difference between the neutron and proton root mean square radii. In the region $1 \leq \eta \leq 2$ one has more preferably proton halo effect as reported in [1]. For the values of $\eta \sim 4-5$, where the tails of the densities are close to that obtained in the quantum mechanical calculations, the halo and skin components of $\Delta r_{n p}$ are found to be approximately equal.

The sensitivity of the partial contributions $\Delta r_{n p}^{\text {skin }} / \Delta r_{n p}$ and $\Delta r_{n p}^{\text {halo }} / \Delta r_{n p}$ on the Skyrme force parametrization is shown in Fig. 3. It is seen from Fig. 3 that the calculated values change of about $20 \%$ for different parametrizations. where we have introduced the notations: $R=\left(R_{n}+R_{p}\right) / 2, a=\left(a_{n}+a_{p}\right) / 2, \Delta_{R}=R_{n}-R_{p}$, and $\Delta_{a}=a_{n}-a_{p}$, and $\kappa_{j}(\xi)$ are the generalized Fermi integrals (see Appendix A).

In Fig. 1 the partial contributions to the neutron-skin thickness from the skin effect, $\Delta r_{n p}^{s k i n} / \Delta r_{n p}$, and the halo effect, $\Delta r_{n p}^{\text {halo }} / \Delta r_{n p}$, are shown. One can see from Fig. 1 that the halo partial contribution increases and the skin partial contribution decreases with the increase of parameter $\eta$. Close to the semiclassical approach region, $1 \leq \eta \leq 2$, the partial contributions from the halo effect are negative and therefore the partial contributions from the skin effect are greater than the unity. The reason for that is the high value of the parameter $\xi$ for the asymmetric Fermi function (Fig. 2), so the densities fall off too quickly in the outer surface.

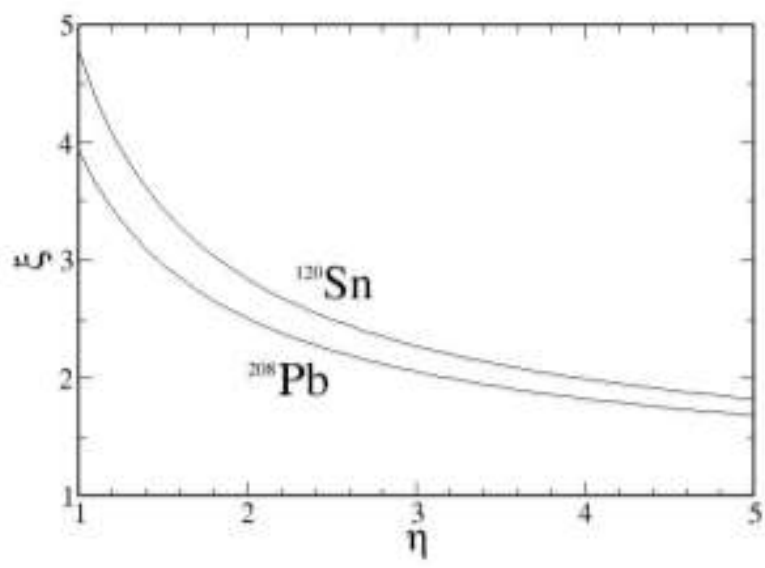

Fig. 2. Parameter $\xi$ versus $\eta$. The calculations have been performed for nuclei ${ }^{120} \mathrm{Sn}$ and ${ }^{208} \mathrm{~Pb}$ using $\mathrm{SkM}$ parametrization.

The dependence of $\Delta r_{n p}$ on the asymmetry parameter $X$ together with the experimental data [7] are shown in Fig. 4. As it is seen from the figure the calculation with the "quantum mechanical" value $\eta=4$ (solid curve), in general, agrees better with the experimental data than for $\eta=2$ (dashed curve). The obtained dependencies are approximately linear for the considered values of isotopic asymmetry parameter $X$.

In Fig. 5 the analogous calculations for tin isotopes together with the experimental data are shown as a function of mass number. Similarly to the previous cases, the calculation with $\eta=4$ describes the experimental data better than the one with $\eta=2$. 


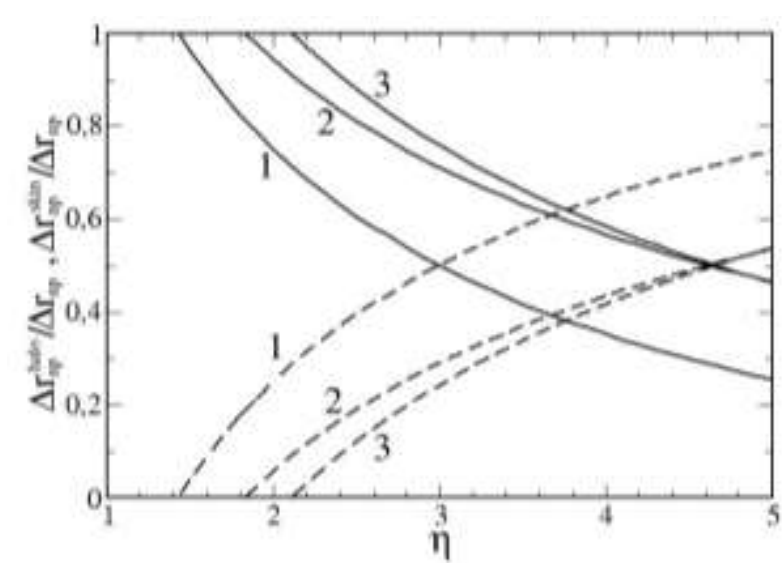

Fig. 3. The partial contribution to the isotopic shift of radii $\Delta r_{n p}$ from the skin effect, $\Delta r_{n p}^{s k i n} / \Delta r_{n p}$ (solid lines) and the halo effect, $\Delta r_{n p}^{\text {halo }} / \Delta r_{n p}$ (dashed lines) versus the parameter $\eta$. The calculations have been performed for ${ }^{120} \mathrm{Sn}$ with the following Skyrme force parametrizations: 1 SIII; 2 - SkM; 3 - SLy230b.

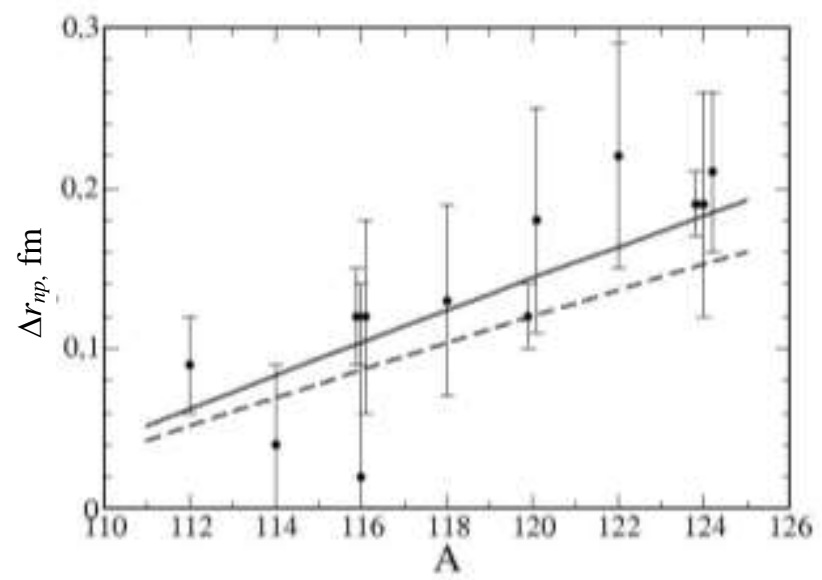

Fig. 5. Difference $\Delta r_{n p}$ between the rms radii of the neutron and proton distributions as a function of $A$ for $\mathrm{Sn}$ isotopes. The solid and dashed lines are calculations with $\eta=4$ and 2 for SkM force. The experimental data are taken from Ref. [7].

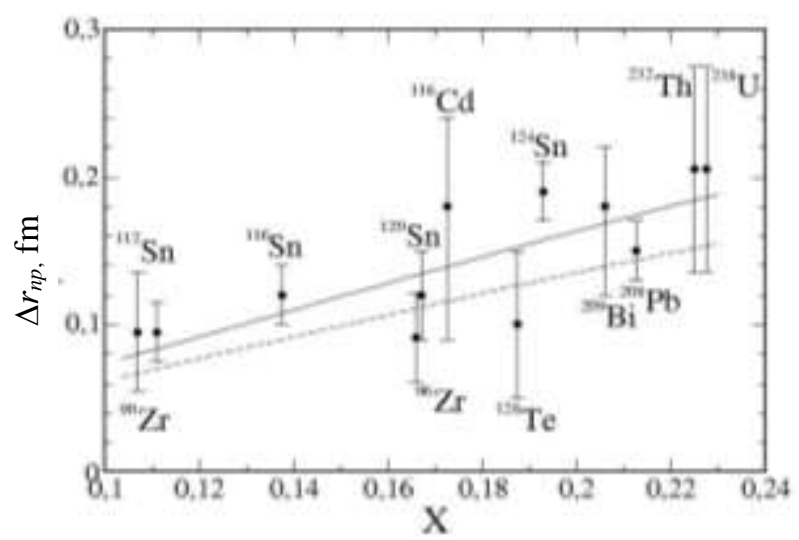

Fig. 4. Difference $\Delta r_{n p}$ between the rms radii of the neutron and proton distributions as a function of $X=(N-Z) / A$. The solid and dashed lines are calculations with $\eta=4$ and 2 for SkM parametrization of the Skyrme force. The experimental data are taken from Ref. [7].

The neutron-to-proton density ratio in the peripheral region with respect to the bulk value is described by the peripheral halo factor [8]. Following $[7,8]$ we will also use the theoretical estimation of the halo factor as

$$
f_{\text {theor }}^{\text {halo }}(r) \approx \frac{\rho_{n}(r)}{\rho_{p}(r)} \frac{Z}{N} .
$$

In Fig. 6 we show the calculations of the theoretical halo factor for two values of the parameter $\eta$ together with the experimental data [7]. As in the previous figures the solid lines correspond to the calculation with $\eta=4$ and dashed lines correspond to $\eta=2$.
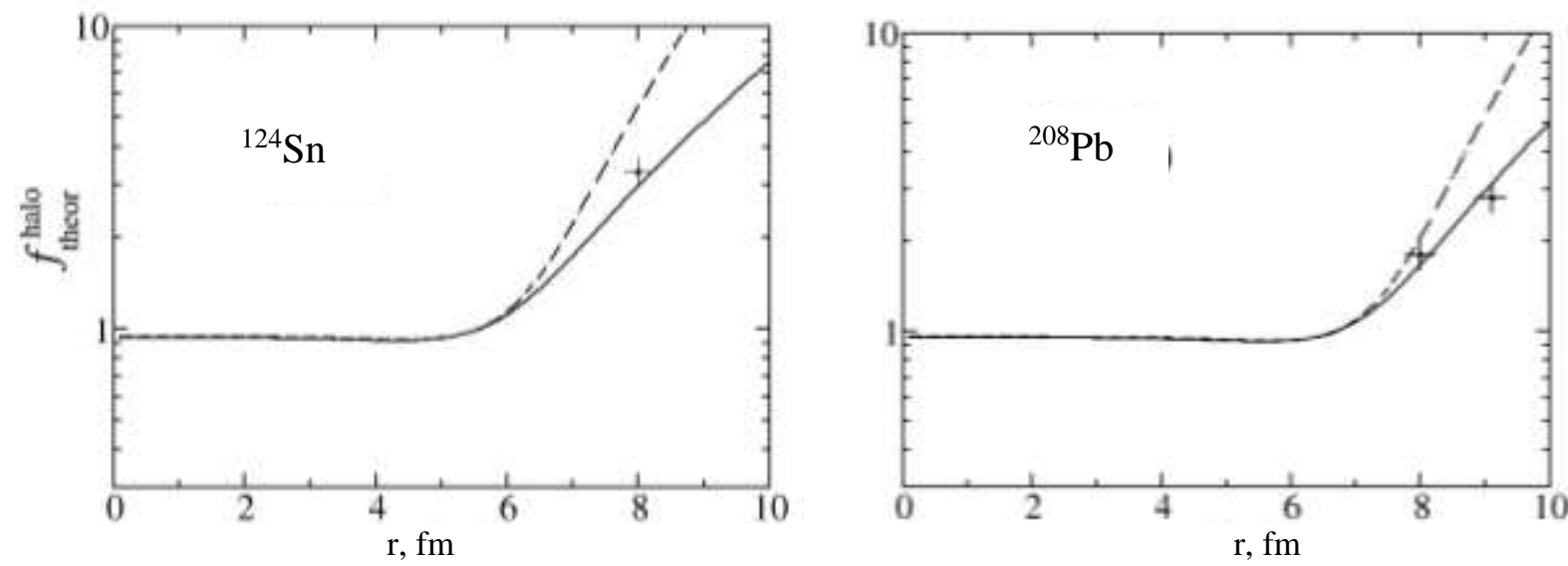

Fig. 6. The halo factor in ${ }^{124} \mathrm{Sn}$ and ${ }^{208} \mathrm{~Pb}$ as a function of the distance from the center of the nucleus calculated using the Eq. (16). The values of the halo factor deduced from experiment are marked by crosses [7]. 
The left panel is the case for tin isotope ${ }^{124} \mathrm{Sn}$, and the right panel is for ${ }^{208} \mathrm{~Pb}$. It is seen from Fig. 6 that the calculation with $\eta=4$ describes the experimental data better than the one with $\eta=2$.

It should be noted here that for all calculations for nuclei with mass numbers $A$ from 90 to 238 the leptodermous parameter $a / R$ does not exceed 0.1 .

\section{Conclusions}

We have considered the neutron skin and halo effects for medium and heavy nuclei within the extended Thomas - Fermi approximation using effective Skyrme-like forces and the direct variational method. The nucleon densities $\rho_{p}(\boldsymbol{r})$ and $\rho_{n}(\boldsymbol{r})$ are generated by the profile functions which are obtained by the requirement that the energy of the nucleus should be stationary with respect to variations of these profiles.

Using the leptodermous properties of the profile nucleon densities $\rho_{p}(\boldsymbol{r})$ and $\rho_{n}(\boldsymbol{r})$, we have obtained the analytical expression for the isovector shift of the rms radii $\Delta r_{n p}$ as a superposition of the two terms. The first one, $\Delta r_{n p}^{s k i n}$, describes the neutron, skin-type" distribution and the second one, $\Delta r_{n p}^{\text {halo }}$, describes the neutron "halo-type" distribution.

Numerical calculations show that the partial contributions to the neutron-skin thickness from the skin effect, $\Delta r_{n p}^{\text {skin }} / \Delta r_{n p}$, and the halo effect, $\Delta r_{n p}^{\text {halo }} / \Delta r_{n p}$, depend on the parameter $\eta$. With the increase of $\eta$ from 2 to $\eta=4$ the partial contributions to the neutron-skin thickness from the skin effect decreases from $100 \%$ to about of $50 \%$ and the halo component increases from zero to about of $50 \%$.

The calculated isovector shift of the rms radii $\Delta r_{n p}$ and halo factor $f_{\text {theor }}^{\text {halo }}(r)$ give satisfactory description for the corresponding experimental data at $\eta=4$. In this case we have superposition of the neutron skin and halo effects with approximately equal contributions.

We would like to thank Prof. V. M. Kolomietz for stimulating our interest to the subject of the article and for useful discussions.

\section{Appendix A}

In this Appendix, we will consider the rms radius for nucleons as

where

$$
\sqrt{r_{q}^{2}}=\sqrt{\frac{\int d \boldsymbol{r} r^{2} \rho_{q}(r)}{\int d \boldsymbol{r} \rho_{q}(r)}}=\sqrt{\frac{I_{4, q}}{I_{2, q}}}
$$

$$
I_{n, q}=\int_{0}^{\infty} d r r^{n} \rho_{q}(r)
$$

Within the leptodermous approximation $a_{q} / R_{q} \ll 1$ one has

$$
\begin{gathered}
I_{2, q} \simeq \frac{R_{q}^{3}}{3}\left(1+3 \kappa_{0}(\xi) \frac{a_{q}}{R_{q}}+6 \kappa_{1}(\xi)\left(\frac{a_{q}}{R_{q}}\right)^{2}+3 \kappa_{2}(\xi)\left(\frac{a_{q}}{R_{q}}\right)^{3}\right), \\
I_{4, q} \simeq \frac{R_{q}^{5}}{5}\left(1+5 \kappa_{0}(\xi) \frac{a_{q}}{R_{q}}+20 \kappa_{1}(\xi)\left(\frac{a_{q}}{R_{q}}\right)^{2}+30 \kappa_{2}(\xi)\left(\frac{a_{q}}{R_{q}}\right)^{3}+20 \kappa_{3}(\xi)\left(\frac{a_{q}}{R_{q}}\right)^{4}+5 \kappa_{4}(\xi)\left(\frac{a_{q}}{R_{q}}\right)^{5}\right),
\end{gathered}
$$

where $\kappa_{j}(\xi)$ are the generalized Fermi integrals derived in Ref. [5]

$$
\kappa_{j}(\xi)=\int_{0}^{\infty} d x x^{j}\left[\left(1+e^{x}\right)^{-\xi}-(-1)^{j}\left(1-\left(1+e^{-x}\right)^{-\xi}\right)\right] .
$$

Inserting Eqs. (A3) and (A4) into the Eq. (A1) one gets

$$
\sqrt{r_{q}^{2}} \simeq \sqrt{\frac{3}{5}} R_{q}\left\{1+\kappa_{0}(\xi) \frac{a_{q}}{R_{q}}-\frac{7}{2}\left(\kappa_{0}^{2}(\xi)-2 \kappa_{1}(\xi)\right)\left(\frac{a_{q}}{R_{q}}\right)^{2}+\frac{1}{6}\left(75 \kappa_{0}^{3}(\xi)-204 \kappa_{0}(\xi) \kappa_{1}(\xi)+81 \kappa_{2}(\xi)\right)\left(\frac{a_{q}}{R_{q}}\right)^{3}\right\} .
$$


From Eq. (A6) the isovector shift of the rms radii $\Delta r_{n p}$ reads

$$
\begin{aligned}
\Delta r_{n p}=\sqrt{r_{n}^{2}}-\sqrt{r_{p}^{2}} \simeq \sqrt{\frac{3}{5}}\left\{R_{n}-R_{p}+\kappa_{0}(\xi)\left(a_{n}-a_{p}\right)-\frac{7}{2}\left(\kappa_{0}^{2}(\xi)-2 \kappa_{1}(\xi)\right)\left(\frac{a_{n}^{2}}{R_{n}}-\frac{a_{p}^{2}}{R_{p}}\right)+\right. \\
\left.+\frac{1}{6}\left(75 \kappa_{0}^{3}(\xi)-204 \kappa_{0}(\xi) \kappa_{1}(\xi)+81 \kappa_{2}(\xi)\right)\left(\frac{a_{n}^{3}}{R_{n}^{2}}-\frac{a_{p}^{3}}{R_{p}^{2}}\right)\right\} .
\end{aligned}
$$

One can rewrite last terms of Eq. (A7) using the relations

$$
\begin{aligned}
& \frac{a_{n}^{2}}{R_{n}}-\frac{a_{p}^{2}}{R_{p}} \simeq-\left(\frac{a_{n}+a_{p}}{R_{n}+R_{p}}\right)^{2}\left(R_{n}-R_{p}\right)+2 \frac{a_{n}+a_{p}}{R_{n}+R_{p}}\left(a_{n}-a_{p}\right), \\
& \frac{a_{n}^{3}}{R_{n}^{2}}-\frac{a_{p}^{3}}{R_{p}^{2}} \simeq-2\left(\frac{a_{n}+a_{p}}{R_{n}+R_{p}}\right)^{3}\left(R_{n}-R_{p}\right)+3\left(\frac{a_{n}+a_{p}}{R_{n}+R_{p}}\right)^{2}\left(a_{n}-a_{p}\right) .
\end{aligned}
$$

Finally, we have

$$
\begin{aligned}
& \Delta r_{n p} \simeq \sqrt{\frac{3}{5}}\left\{1+\frac{7}{2}\left(\kappa_{0}^{2}(\xi)-2 \kappa_{1}(\xi)\right)\left(\frac{a_{n}+a_{p}}{R_{n}+R_{p}}\right)^{2}-\frac{1}{3}\left(75 \kappa_{0}^{3}(\xi)-204 \kappa_{0}(\xi) \kappa_{1}(\xi)+81 \kappa_{2}(\xi)\right)\left(\frac{a_{n}+a_{p}}{R_{n}+R_{p}}\right)^{3}\right\}\left(R_{n}-R_{p}\right)+ \\
& +\sqrt{\frac{3}{5}}\left\{\kappa_{0}(\xi)-7\left(\kappa_{0}^{2}(\xi)-2 \kappa_{1}(\xi)\right) \frac{a_{n}+a_{p}}{R_{n}+R_{p}}+\frac{1}{2}\left(75 \kappa_{0}^{3}(\xi)-204 \kappa_{0}(\xi) \kappa_{1}(\xi)+81 \kappa_{2}(\xi)\right)\left(\frac{a_{n}+a_{p}}{R_{n}+R_{p}}\right)^{2}\right\}\left(a_{n}-a_{p}\right) . \quad \text { (A10) }
\end{aligned}
$$

\section{REFERENCES}

1. Warda M., Viñas X., Roca-Maza X., Centelles M. Analysis of bulk and surface contributions in the neutron skin of nuclei // Phys. Rev. - 2010. - Vol. C81. P. 054309.

2. Kirzhnitz D.A. Field Theoretical Methods in Many Body Systems. - London: Pergamon, 1967.

3. Brack M., Guet C., Håkansson H.-B. Selfconsistent semiclassical description of average nuclear properties - a link between microscopic and macroscopic models // Phys. Rep. - 1985. - Vol. 123. - P. 275 - 374.

4. Kolomietz V.M. Nuclear Fermi-Liquid. - Kyiv: Nauk. Dumka, 2009. - 414 p. (Rus)
5. Kolomietz V.M., Sanzhur A.I. Equation of state and symmetry energy within the stability valley // Eur. Phys. J. - 2008. - Vol. A38. - P. 345 - 354.

6. Mizutori S., Dobaczewski J., Lalazissis G.A. et al. Nuclear skins and halos in the mean-field theory // Phys. Rev. - 2000. - Vol C 61. - P. 044326.

7. Trzcińska A., Jastrzébsky J., Lubiński P. et al. Neutron density distributions deduced from antiprotonic atoms // Phys. Rev. Lett. - 2001. - Vol. 87. - P. 082501.

8. Bugg W.M., Condo G.T., Hart E.L. et al. Evidence for a neutron halo in heavy nuclei from antiproton absorption // Phys. Rev. Lett. - 1973. - Vol. 31. - P. 475.

\section{С. В. Лук'янов, А. І. Санжур}

Інститут ядерних досліджень НАН Украӥни, Київ

\section{НЕЙТРОННІ ШКІРА ТА ГАЛО В СЕРЕДНІХ ТА ВАЖКИХ ЯДРАХ У РАМКАХ РОЗШИРЕНОГО НАБЛИЖЕННЯ ТОМАСА - ФЕРМІ}

У рамках розширеного наближення Томаса - Фермі розраховано нейтронні розподіли типу шкіри та гало для середніх та важких ядер. Розрахунки проводились із застосуванням прямого варіаційного методу для ефективних сил Скірма. Отримано аналітичні вирази для ізовекторного зсуву середньоквадратичних радіусів $\Delta r_{n p}$ у вигляді суми членів, що описують нейтронні шкіру та гало. Знайдено, що вклади від членів, які описують шкіру та гало, у $\Delta r_{n p}$ приблизно рівні.

Ключові слова: нейтронна шкіра, нейтронне гало, теорія Томаса - Фермі, варіаційний метод, сили Скірма. 


\section{С. В. Лукьянов, А. И. Санжур}

Институт ядерных исследований НАН Украины, Киев

\section{НЕЙТРОННЫЕ КОЖА И ГАЛО В СРЕДНИХ И ТЯЖЕЛЫХ ЯДРАХ В РАМКАХ РАСШИРЕННОГО ПРИБЛИЖЕНИЯ ТОМАСА - ФЕРМИ}

В рамках расширенного приближения Томаса - Ферми рассчитаны нейтронные распределения типа кожи и гало для средних и тяжелых ядер. Расчеты проводились с использованием прямого вариационного метода для эффективных сил Скирма. Получено аналитические выражения для изовекторного сдвига среднеквадратических радиусов $\Delta r_{n p}$ в виде суммы членов, описывающих нейтронные кожу и гало. Найдено, что вклады от членов, описывающих кожу и гало, в $\Delta r_{n p}$ приблизительно равны.

Ключевые слова: нейтронная кожа, нейтронное гало, теория Томаса - Ферми, вариационный метод, силы Скирма.

Надійшла 02.09.2015

Received 02.09.2015 